\title{
The Saturn rings origin: contribution of electromagnetism (to the unified theory of the origin of planetary rings)
}

\author{
Vladimir V. Cherny (Tchernyi) \\ Modern Science Institute at SAIBR, Moscow, Russia
}

Email address:

chernyv@mail.ru

\section{To cite this article:}

Vladimir V. Cherny (Tchernyi). The Saturn Rings Origin: Contribution of Electromagnetism (to the Unified Theory of the Origin of Planetary Rings). American Journal of Astronomy and Astrophysics. Vol. 1, No. 2, 2013, pp. 15-22.

doi: 10.11648/j.ajaa.20130102.11

\begin{abstract}
Electromagnetic modeling and experimental data observation for Saturn's rings points to the conjecture that the particles constituting the rings may be superconductive. We also argue that the rings could be originated from the protoplanetary cloud of particles if particles are superconducting. The rings system emerges some time after the magnetic field of the planet is being formed. For example it can be a result of the interaction of the superconducting carbon doped ice particles of the protoplanetary cloud with the nonuniform magnetic field. After a transition period as 1000 years or more, all Keplerian orbits of the particles form a sombrero disc in the plane of the magnetic equator where there is a minimum of magnetic energy. The gravitational resonances and other interactions also play an important role and they help bringing the order to the system of rings and gaps. Electromagnetism and superconductivity helps us to understand why the rings appear only for planets if they are located outside the asteroid belt that have a magnetic field and where the temperature is low enough, such as Jupiter, Saturn, Uranus and Neptune. We end up with a unified theory of the formation of planetary rings. The presented model allows enrich the well-known theories that treat gravitational, mechanical, gas-plasma, dusty plasma and magnetohydrodynamic interactions in a consistent way.
\end{abstract}

Keywords: Saturn System, Saturn Rings Origin, Superconductivity of Saturn Rings, Origin of Planetary Rings, Planetary Rings Electromagnetism, Planetary Rings Superconductivity, Space Electromagnetism, Space Superconductivity

\section{Introduction}

Presently there are two versions of the Saturn rings origin: rings were originated from the debris of the asteroid-type body destroyed nearby the planet by gravity and centrifugal forces, or the system of the rings was formed from the particles of the protoplanetary cloud that initially surrounded Saturn.

We attempt to show why the second model could work. Namely, the rings appeared as a result of the electromagnetic interaction of iced particles of the protoplanetary cloud with the magnetic field of Saturn. This could happen if particles possess superconductivity as Saturn has a magnetic field and in its vicinity the temperature is low enough, $70-110 \mathrm{~K}$.

The proposed model further suggests that the ring particles are relics of the early days of the Solar system, and the particles were never subjected to excessive heating and coalescence. We hope the presented model would enrich classical theories of the rings origin such as gravitational, mechanical, dusty plasma, gas-plasma, and magnetohydrodynamic interactions in a consistent way.

G. Galileo (1610), Ch. Huygens (1665), G. Cassini (1671), W. and G. Bond (1848), J.K. Maxwell (1859), S. Kovalevskaya (1874) and others studied the Saturn rings nature [1-21, 26-39]. Different ring systems are morphologically quite distinct but all are shaped by a few common processes. In fact, orbital resonances between satellites, moons and ring particles play an important role in forming a specific structure of the rings and gaps and enhancing the influence of the satellites. An experimental data confirmed importance of magnetohydrodynamic, gasplasma, dusty plasma and electromagnetic phenomena for the rings nature.

For the first time the role of superconductivity for the origin of Saturn rings was presented by A.Yu. Pospelov and V.V. Tchernyi in 1995 and further [26-30], published $[31,36]$ and discussed in detail [26-39]. Also we expect the proposed model that assumes existence of a 
superconducting fraction of the particles forming the Saturn rings would allow enhancing classical theories of the planetary rings.

Let's go to the short history of the problem. H. Kamerlingh Onnes discovered superconductivity in 1911. In 1933 W. Meissner and R. Ochsenfeld found that a superconducting material will repel a magnetic field. The high-temperature superconductivity was discovered by J.G. Bednorz and K.A. Muller in 1986 [22]. Superconductivity of ice was experimentally demonstrated by A.N. Babushkin et al in 1986 [23]. Superconductivity of $\mathrm{C}_{36}$ has been conjectured in 1998 [24]. So, even 7\% of a glassy carbon composition of Saturn rings may contribute to its superconductivity. In 2011 scientists lead by Deutscher G. demonstrated how a superconductor disc is trapped in a surrounding magnetic field, a phenomenon called "Quantum Locking" and "Quantum Levitation" [40]. By using the exceptional large scale superconductors they are able to demonstrate a quantum effect that was never seen and demonstrated this way. They showed how a disc frozen with liquid nitrogen can be made to hover over a magnet in any position.

\section{Electromagnetic Modeling of the Origin of Saturn Rings [32-35, 37-39]}

\subsection{Origin of the Rings from the Iced Particles of the Protoplanetary Cloud}

Prior to emergence of the Saturn magnetic field, all the particles within the protoplanetary cloud are located on the Keplerian orbits exhibiting a balance of the force of gravity and the centrifugal force. With emergence of the Saturn magnetic field the superconducting particles of the protoplanetary cloud begin to demonstrate an ideal diamagnetism (Meissner-Ochsenfeld phenomenon). Particles begin to interact with the magnetic field and all the particles become involved in an additional azimuthorbital motion. Let's estimate the result of this motion.

If the magnetic field of the planet is $\mathrm{H}$ and the planetary magnetic moment is $\mu$, then the magnetic field at any particular point within the protoplanetary cloud located at the distance $\mathbf{r}$ can be presented as:

$$
\mathbf{H}=\frac{3 \cdot \mathbf{r} \cdot(\mathbf{r}, \boldsymbol{\mu})}{r^{5}}-\frac{\boldsymbol{\mu}}{r^{3}}
$$

Then the superconducting sphere of the radius $\mathrm{R}$ located within the protoplanetary cloud acquires the magnetic moment

$$
\mathbf{M}=-R^{3} \cdot \mathbf{H}
$$

The energy of the superconductor in the magnetic field is:

$$
U_{H}=-(\mathbf{M}, \mathbf{H})=R^{3} \cdot H^{2}
$$

Placing the origin of the coordinates at the center of the planet and directing the $\mathrm{z}$ axis along the magnetic moment of the planet (orthogonal to equator), the expression for magnetic energy then becomes:

$$
U_{H}=\frac{R^{3} \mu^{2}}{r^{6}}\left(3 \cos ^{2} \theta+1\right) .
$$

Here $\theta$ - the angle between the vector $r$ and the axis $\mathrm{z}$. It can be seen from the expression (2.4) that the magnetic energy of the superconducting particle has a minimum value when the radius-vector $r$ (the position of the superconducting particle) is in a plane of the magnetic equator and is perpendicular to the axis $\mathrm{z}(\cos \theta=0)$. Consider now only one particle. Evidently its azimuthorbital direction trajectory (orbit) can only be disturbed by the magnetic field. However in case of a significant amount of particles forming the protoplanetary cloud, after a transient time estimated as 1000 years or more, collisions between particles will compensate their azimuth-orbital movements, and as a result all orbits of the particles of the protoplanetary cloud should come together to the magnetic equator plane and create highly flattening disc around the planet. Within the disc of the rings all particles will become located on the Keplerian orbit where there is a balance of gravity, centrifugal and electromagnetic forces. At the same time orbital resonances (due to a gravity force) between satellites, moons and the ring particles would play an important role in forming a specific structure of the rings and gaps.

\subsection{Particle Repelling and Collision within the Ring Width}

Let's define the energy of the interaction of two superconducting particles with the magnetic moments $\boldsymbol{\mu}_{1 z}$ and $\mu_{2 \Sigma}$ located at positions $\mathrm{r}_{1}$ and $\mathrm{r}_{2}$, respectively as:

$$
U=-\boldsymbol{\mu}_{1} \mathbf{H}_{2},
$$

The magnetic field $\mathbf{H}_{2}$ induced by the magnetic moment $\boldsymbol{\mu}_{2}$ can be presented as

$$
\mathbf{H}_{2}=\frac{3\left(\mathbf{r}_{1}-\mathbf{r}_{2}\right)\left(\boldsymbol{\mu}_{2}\left(\mathbf{r}_{1}-\mathbf{r}_{2}\right)\right)}{\left|\mathbf{r}_{1}-\mathbf{r}_{2}\right|^{5}}-\frac{\boldsymbol{\mu}_{2}}{\left|\mathbf{r}_{1}-\mathbf{r}_{2}\right|^{3}}
$$

If the particle with the magnetic moment $\mu_{2}$ is placed at the origin $\left(r_{2}=0\right)$ then the expression for the energy of the interaction of two particles (2.5) will read:

$$
U=-\frac{3\left(\boldsymbol{\mu}_{1} \mathbf{r}_{1}\right)\left(\boldsymbol{\mu}_{2} \mathbf{r}_{1}\right)}{\left|\mathbf{r}_{1}\right|^{5}}+\frac{\boldsymbol{\mu}_{1} \boldsymbol{\mu}_{2}}{\left|\mathbf{r}_{1}\right|^{3}}
$$

The planetary magnetic field in the plane of the Saturn rings coincides with the planet rotation axis. If the axis $\mathrm{z}$ is directed along the rotation axis of the planet, then the magnetic moment of the particles will be also directed along z. In cylindrical coordinate system $(\rho, \varphi, z)(2.7)$ is: 


$$
\begin{gathered}
U=-\left(\frac{3 z^{2}}{\left(\rho^{2}+z^{2}\right)^{5 / 2}}-\frac{1}{\left(\rho^{2}+z^{2}\right)^{3 / 2}}\right) \mu_{1 z} \mu_{2 z}= \\
=\frac{\rho^{2}-2 z^{2}}{\left(\rho^{2}+z^{2}\right)^{5 / 2}} \mu_{1 z} \mu_{2 z}
\end{gathered}
$$

From (2.8) we can estimate an interaction of two superconducting particles for two different cases. The first one is when two particles located in the same plane within the sombrero of the rings $(\mathrm{z}=0)$, and the second situation is when two particles are located on the different planes but on the same axis $(\rho=0)$. For the particles with the magnetic moments $\mu_{1 z}$ and $\mu_{2 z}$ located on the same plane, $\mathrm{z}=0$, we get the interaction energy as:

$$
U=\frac{\mu_{1 z} \mu_{2 z}}{\rho^{3}}
$$

From (2.9) it follows that both particles will repel each other and they will maintain a separate distance between them. This result has been confirmed by the data of Cassini mission: the particles are separated. If particles are located on the same axis but on different planes, the expression for the interaction energy is:

$$
U=-\frac{\mu_{1 z} \mu_{2 z}}{|z|^{3}},
$$

Now both particles could attract each other; they could even collide or stick together and form bigger clusters or lumps of ice. This process has an experimental conformation by the Cassini mission. From the data of the Cassini mission it follows that the particles within the thickness of the rings can collide or even stick together and create bigger clusters of ice. Then, in the following process, particles of 50 meters or more in diameter can be shattered into smaller pieces by a combined action of gravity and centrifugal force.

\section{Discussion of Experimental Observations [26-31, 36-39]}

\subsection{Thin Width and Sharp Edges of the Rings}

Similar to the iron magnetic particles that create dense and rarefied regions in the nonuniform magnetic field, superconducting particles also form bands looking from outside like a system of rings. Superconducting particles collapse into the stable system of rings as a result of their exchange between the areas of the gradient of magnetic field within the plane of the magnetic equator with the force: $\mathrm{F}=-\mu \mathrm{dH} / \mathrm{dz}$, where $\mu-$ the magnetic moment of the particle, $\mathrm{dH} / \mathrm{dz}$ - the gradient of the magnetic field along the $\mathrm{z}$ axis of the magnetic dipole. The force of the diamagnetic expulsion forms sharp edges of the ring: $\mathrm{F}=$ $\mu \mathrm{dH} / \mathrm{dy}$, where $\mathrm{dH} / \mathrm{dy}$ - the gradient of the magnetic field along the radius of the ring. The accidental break in the ring will be stabilized by the force of the diamagnetic expulsion $\mathrm{F}=-\mu \mathrm{dH} / \mathrm{dx}$, where $\mathrm{dH} / \mathrm{dx}$ - gradient of the magnetic field in the tangential direction. The image of the magnetic field line deformation measured for the ring $\boldsymbol{F}$ by the Pioneer mission looks like the image of the magnetic field expulsed from the ring. It is of the same nature as for the well-know case of a small superconducting ceramic sample pushing out its own internal magnetic field, when exposed to a liquid nitrogen temperature.

\subsection{Planetary Radial Dust Flow}

Superconducting material is characterized by the London's penetration depth $\lambda_{\mathrm{L}}$ of the magnetic field. For particles of size comparable with the London's penetration depth the influence of the magnetic field on superconductivity becomes appreciable. Smaller particles do not couple to the planetary magnetic field because they lose their superconductivity due to their small size. The dynamics of these particles is different from the dynamics of the particles with larger size, $>2 \lambda_{\mathrm{L}}$. Small particles will fall down to the planet due to gravity. Thus, existence of a radial planetary dust flux composed of submicron's size particles is related to a lack of superconductivity of the ring particles due to their small size. It is also possible for the particles to lose their superconductivity by collisions and by magnetic field fluctuations.

\subsection{The Azimuthal Brightness of the Saturn A Ring}

Present understanding of this phenomenon is based on: a) an assumption of a synchronous rotation of the ring's particles with their asymmetrical form as extended ellipsoids directed under a small angle to the orbit; b) existence of an asymmetrical albedo of the surface. Consider now our model for this phenomenon. If the superconductor is placed in the magnetic field, a magnetic moment directed oppositely to the external field is induced. The matter is magnetized not along the external magnetic field but in the opposite direction. A superconductive rings particle in the form of the rod attempts to align itself perpendicularly to the magnetic field lines. It is a known fact from science of ice [25] that growing snowflakes at the temperature below $-22^{0} \mathrm{C}$ take the form of prisms. Thus, the prism of the superconducting ice particle will be oriented perpendicularly to the field lines of the poloidal and toroidal components of the magnetic fields of the Saturn. It becomes now clear that the variable azimutal brightness of the Saturn's rings system $A$ is related to the orientation of the elongated ellipsoid of the superconducting particles versus the direction of the planetary magnetic field.

\subsection{Spokes in the $B$ rings system}

Just as any wheel spokes, the spokes of the rings are aligned almost radially. The size of the spokes is about 104 $\mathrm{km}$ along the radius and about $103 \mathrm{~km}$ along the orbit of 
the rings. The matter of the spokes consists of micron and submicron size particles. There were many attempts to explain the nature of these spokes. Generally, all the models are based on the action of the force of gravity. Nevertheless a different idea was put forward that the nature of rotating spokes perhaps is related to the electromagnetic force. Analysis of the spectral radiation power of spokes provides a specific periodicity about $640.6 \pm 3.5$ min which almost coincides with the period of rotation of the magnetic field of Saturn (639.4 $\mathrm{min}$ ). Moreover a strong correlation of maxima and minima of activity of spokes with the spectral magnetic longitudes is connected to presence or absence of the radiation of Saturn's Kilometric Radiation (SKR). It enhances the assumption of the dependence of the spoke dynamics on the magnetic field of Saturn and testifies to the presence of large-scale anomalies in the magnetic field of Saturn. We can add the following explanation of it.

Superconducting ice particles of the ring matter are orbiting in accordance with the Kepler's law and have their own speed on each orbit. Further, a magnetic field of Saturn has its own anomalies along some radial direction from the planet. When the particles enter into this anomalous region, the diamagnetic expulsion force that is applied to the particles changes its value. The particles then begin to change their orbit. For the significant number of participating particles, for the external observer, this process appears as the turbulent cloud stretched along the radius in the form of spokes. After passing anomaly, particles return to their prior orbit and the common appearance of the rings is recovered.

\subsection{High Reflection and Low Brightness of the Rings Particles in the Radiofrequency Range}

This also can be explained by the superconductivity of the ice particles. Discovery in 1973 of the strong radartracking reflection from the rings of Saturn was surprising. It turned out that the rings of the Saturn actually have the greatest radar-tracking section among all bodies of the Solar system. It was explained by assuming a metallic nature of the particles. The data of the Voyager excludes this possibility. The disk of superconducting particles completely reflects radiation with frequencies below $10^{11}$ $\mathrm{Hz}$ and poorly reflects radiation with higher frequencies, as in the case of a superconductor. The superconductor practically has no electric resistance up to frequencies of $100 \mathrm{MHz}$. A threshold is about $100 \mathrm{GHz}$ and above. From Fig. 1 we can see the sharp change of resistance. It may be caused by quantum phenomena in this range. Consequently it produces a specific dependence of the brightness.

\subsection{Intrinsic Wide Band Pulse Radiation of the Rings}

Data of Voyager have shown that the rings radiate intrinsic wide band pulse radiation within the $20 \mathrm{KHz}$ 40,2 MHz. These waves probably are the result of an interaction of charged particles with the particles of ice and friction of ice particles when the co-striking occurs. These incidental radio discharges are called Saturn's Electrostatic Discharges (SED). The average period of SED is well defined by Voyager-1, -2 in between 10 hours $10 \pm 5 \mathrm{~min}$ and 10 hours $11 \pm 5 \mathrm{~min}$. If the ring has a source of SED then the area of this source can be located at the distance of $107,990-109,000 \mathrm{~km}$ from the planet according to the measured periodicity.

Experimental data for SKR, SED and spokes activity specify the electromagnetic coupling between the planetary ring system and the magnetosphere of the planet. As it goes
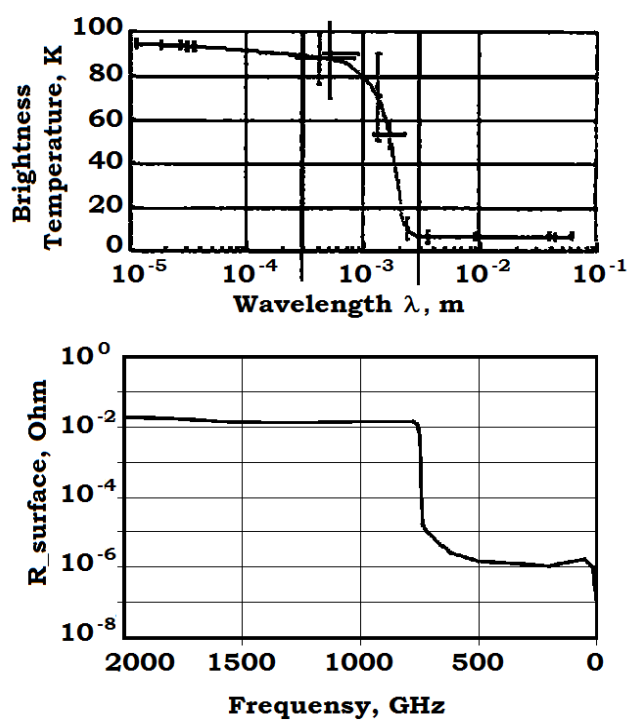

Fig.1. The top picture is the spectral dependence of the brightness temperature of the rings. Practically, we have a transition from blackbody radiation to almost total reflection is observed [31, 36]. The bottom diagram is dependence of the surface resistance of the superconductor on frequency for $\mathrm{Nb}$ at $T=2 K$ [Brinkmann R., Dohlus M., Trines D., Novokhatski A., Timm M., Weiland T., Hulsmann P., Rieck C.T., Scharnberg K., Schmuser P. March 2000. Terahertz Wakefields in the Superconducting Cavities of the TESLA-FEL Linac. Tesla Reports].

from consideration of the presented electromagnetic model, for superconducting particles approaching distance about $10^{-8} \mathrm{~m}$ or if they have a point contact, a superconducting transition can occur, as electrons can be tunneled through the gap. Consequently, this type of superconducting weak link begins to generate electromagnetic radiation - a nonstationary Josephson phenomenon for superconductors. The radiation frequency is proportional to the junction voltage, $v=2 \mathrm{eV} / \mathrm{h}$, where $2 \mathrm{e} / \mathrm{h}=483,6 \mathrm{MHz} / \mu \mathrm{V}$, e is the charge of electron, $\mathrm{h}$ is the Plank constant.

\subsection{Frequency Anomalies of Thermal Radiation of the Rings in the Range of $100 \mu \mathrm{m}-1 \mathrm{~cm}$}

The measured brightness temperature for the short wavelengths is less than the true brightness temperature of the rings and, for the longer wavelengths, the rings look much colder than in the case when the radiation corresponds to their physical temperature. Within the range $100 \mu \mathrm{m}-1 \mathrm{~mm}$ the brightness temperature of the ring (Fig. 
1) sharply falls below the black body characteristics. For the wavelengths longer than $1 \mathrm{~cm}$ a ring behaves as the diffusion screen, reflecting planetary and cold space radiation. The central part of the spectral range $100 \mu \mathrm{m}-1$ $\mathrm{cm}$ is the most sensitive part of this dependence which may contain the important information for the fundamental properties of the substance of the particles. In the superconductor, electrons do not interact with the crystal lattice and do not exchange energy with it. That's why there is no heat transfer from one part of the body into another. Hence, when the substance passes into a superconducting condition, its heat conductivity is lowered. And for temperatures significantly below critical, there are very few ordinary electrons capable of transferring heat.

\subsection{Color Difference in the Small Scale of Rings}

The balance of the three forces determines the position of the superconducting particles in the gravitational and magnetic planetary fields: gravitational force, centrifugal one and magnetic levitation (diamagnetic expulsion), Fig.1. Following our model, we consider a distribution of three particles $(\mathrm{a}, \mathrm{b}, \mathrm{c})$ with equal weights and being on nearby orbits. Let the particles a - entirely of a superconducting pure ice, $b$ - the ice particles with an impurity of clathratehydrates of ammonia or methane $\left(\mathrm{NH}_{3} ; \mathrm{CH}_{4} \mathrm{H}_{2} \mathrm{O}\right), \mathrm{c}-$ ice particles with impurities of sulfur and iron containing silicates $\left(\mathrm{H}_{2} \mathrm{~S}\right)$. Each impurity provides its own contribution to the superconductivity phase and it will determine the color of the particle. The force of a diamagnetic expulsion $\mathrm{F}_{\mathrm{L}}$ depends on the volume of the superconducting phase. Therefore for each of the considered particles the balance of the three forces on the orbit has a different radius.

\subsection{Anomalous Inversed Reflection of Circularly Polarized Microwaves for Wavelengths above $1 \mathrm{~cm}$}

The study of reflection of radiowaves with the wavelength more than $1 \mathrm{~cm}$ from the rings has been made by the ground based radar and by the space probe. The reflection appears rather large and the geometrical albedo is about 0.34 and it has no strong dependence on the wavelength or on the angle of the inclination of the ring's pitch. So the rings are strong depolarizers. That's why in order to get any information from reflections measurements it is necessary to measure a reflected signal of two orthogonal polarizations separately. The reflected portion of the signal of the same polarization as the incoming signal is called the signal of "observed" polarization. The perpendicular component is called a signal of "unobserved" polarization. A difference between these two signals provides information about so the called factor of polarization which indicates polarization properties of the object.

For the planets of the Solar system, a reflected signal of unobserved polarization is usually small. As for the Saturn rings, for the same range of wavelengths and angles of an inclination of the incident wave, the factor of polarization becomes much bigger. It has an explanation based on theory of electromagnetic waves reflection from a superconductor. The superconductor differs significantly from the ideal conductor. It has almost infinite conductivity and it also demonstrates an ideal diamagnetism. In case of a reflection from the superconductive ice particle rings, it means that if the incident wave of the radar signal with a circular polarization has certain chirality, then the same chirality should be for the reflected wave.

\subsection{An Atmosphere of Unknown Origin at the Rings}

The atmosphere of Saturn's rings can originate as a result of the thin balance of forces of gravitational attraction and diamagnetic expulsion of gas molecules. Levitation of gas molecules may be originated as a result of its diamagnetic expulsion from superconducting particles due to induced magnetic moments by a magnetic field of the planet. A similar situation can be observed under laboratory conditions when an atmospheric water steam is precipitated on a substance as a white-frost at the transition point of the substance from the superconducting into a conventional state.

\subsection{Existence of Waves of Density and Bending Waves within the Rings}

The existence of the waves of density and bending waves in the Saturn rings has no complete explanation based only on gravitational phenomena. Let's use our model. Note that the external magnetic field is directed along a free surface of the diamagnetic fluid representing a disk of the rings. Consider a localized deformation of the disc surface at some point of the ring. It can be induced, for example, by fluctuating gravitational forces of Saturn moons or satellites, or due to magnetohydrodynamic, gasplasma and dusty plasma effects. Then a ponderomotive force will be created and applied in the opposite direction to preserve an original disc surface. Therefore the planetary magnetic field enhances the stiffness and stability of the disc surface.

\section{Conclusion}

This paper presents an idea which assumes the rings are formed from the particles of the protoplanetary cloud around the Saturn. By itself, this idea is well-known. However, the model has not been fully developed yet. This paper proposes a novel mechanism how rings could originate from the frozen particles of the protoplanetary cloud after the formation of the magnetic field of the Saturn. But for this type of scenario particles should possess superconductivity. This suggestion makes sense because Saturn has a magnetic field and its surroundings temperature is low enough about $70-100 \mathrm{~K}$.

On the basis of the analytical discussion of these groundbased and space-based experiments it was shown that the 
particles of Saturn's rings may have superconductivity. Therefore, presence of superconducting substance may be possible in the space at the asteroid belt and behind it. Although superconducting particles can form a relatively compact macroscopic structure formation, on short distances however they weakly repel each other in presence of an external magnetic field (resembling a fluidized bed).

At the beginning when idea of superconductivity of the rings particles came to us we decided just report [26-30] and published it $[31,36]$. We remember as Pyotr Kapitsa the Nobel Prize winner (1978) for the work in lowtemperature physics said: "The worst in scientific work is triviality. The most important is not accuracy but novelty. Never miss a chance of publication of new ideas." (Uspekhi Fizicheskih Nauk - Physics-Uspekhi. Advances in Physical Sciences, 1994, Vol. 164, p. 1326).

But resolution of the proposed problem is based on the solution of the task of the electromagnetic interaction of the moving superconductive ice-carbon particles with magnetic field of Saturn. This problem was solved in an article using electromagnetic simulation. Finally, after mathematical treatment of the electromagnetic model we have received demonstration and prove of reality of the suggested scenario of the rings origin [32-35, 37-39].

Similarly to the iron particles that create dense and rarefied regions in the nonuniform magnetic field on the laboratory table, superconducting iced particles in the magnetic field of Saturn also form bands which look from outside like a system of rings. But the difference between these two examples is that the superconducting particles are pushed out the internal magnetic field, it repels them, and that's why rings particles will not stick together. Finally after transitional period of time superconducting iced particles of the protoplanetary cloud would collapse into the stable system of rings within the plane of the magnetic equator as a result of their interaction between the areas of the gradient of magnetic field.

Different ring systems are morphologically quite distinct but all are shaped by a few common processes. In fact orbital resonances between satellites, moons and ring particles play an important role in forming a specific structure of the system of the rings and gaps and enhancing the influence of the satellites as well as the gravitational, mechanical, magnetohydrodynamic, dusty plasma and gasplasma, interactions.

Also superconductivity of the ring particles may reflect the fact that the ring particles are relics of the early days of the Solar system and the particles were never subject to coalescence and heating.

The presented theory of the origin of Saturn's rings from the protoplanetary cloud based on superconductivity of its particles is a direct continuation of the J.K. Maxwell theory (1859). The founder of the theory of electromagnetic waves in his award winning paper on the subject "On the stability of the motion of Saturn's rings", deduces that the rings of the Saturn cannot be solid and the rings could be stable only if they consist of "an indefinite number of unconnected particles orbiting Saturn in much the same way as our Moon orbits the Earth, gravitational forces otherwise would destroy them" [1]. Ground based experiments and the data from Pioneer, Voyager $-1,-2$ and Cassini-Huygens space missions have revealed that the rings are composed of separate pure ice particles and ice particles with carbon and other impurities.

Unfortunately, at Maxwell time there was no knowledge about superconductivity (discovered in 1911) and the force of a diamagnetic expulsion of the magnetic field from the superconductor (discovered in 1933). High temperature superconductivity [22] and superconductivity of ice [23] were discovered in 1986. Superconductivity of $\mathrm{C}_{36}$ has been conjectured in 1998 [24].

An important demonstration happened in 2011 when physicists lead by Deutscher G. has been experimenting with superconductors trapped in a magnetic field to produce "quantum locking" and "quantum levitation [40]: http://www.youtube.com/watch?v=Ws6AAhTw7RA. By using the exceptional large scale superconductors they are able to demonstrate a quantum effect that was never seen and demonstrated this way. They showed how a disc frozen with liquid nitrogen can be made to hover over a magnet in any position. Similarly, it can "fly" over a track at any height or at any angle. It even appeared to defy gravity as it circled underneath the track.

The foregoing electromagnetic modeling and discussion of the experimental data significantly strengthen the model of a formation mechanism of planetary rings due to electromagnetism and superconductivity. The presented theory allows us to extend existing theories of the rings origin such as gravitational, mechanical, magnetohydrodynamics, gas-plasma and dusty plasma interactions in a coherent way without conflict with them.

Electromagnetism and superconductivity helps us understand why the rings appear only for the planets if planets are located outside the asteroid belt that have a magnetic field and where the temperature is low enough. Notice that for the planets located inside the asteroid belt heat destroys superconductivity.

Intriguing fact is that presented above scenario of the rings origin for the Saturn is applicable for the other planets - Jupiter, Uranus and Neptune. As a result, we obtain a unified theory of the origin of the planetary rings. It is applicable for Jupiter, Saturn, Uranus and Neptune as the physical conditions of these planets in general look the same - existence of the magnetic field and low temperature environment.

From the above analysis we come to a conclusion about the need to consider the natural superconductivity in the Solar system space outside the belt of asteroid. It may have a fundamental feature for analyzing data of the Cassini probe and striking parallels to other stars system. The force of a diamagnetic expulsion of the magnetic field from the superconductor may be a driving force for propagation of organic molecules within the interstellar space by electromagnetic means, and organic molecules 
with superconductivity can also be contained within the system of the Saturn rings as it is presented in [41-42].

Also as an outcome we can see that the conclusion made by H. Alfven [5] that the "solar system history as recorded in the Saturn rings structure" becomes a physical reality because the proposed electromagnetic model of the Saturn rings origin due to the superconductivity of the protoplanetary cloud particles provides a contribution to it.

\section{Acknowledgments}

The author would like to express greatest thanks to Evgeny P. Bazhanov, Tom V. Zaitsev, Andrew Yu. Pospelov, Evgeny V. Chensky, Anri A. Rukhadze, Valery A. Miliaev, Valery A. Dybov (Moscow, Russia), John R. Whinnery and Ture K. Gustafson at UC Berkeley, Jin Au Kong at MIT Cambridge, Essam A. Marouf at San Jose State University, CA, James F. Spann, Robert B. Sheldon and Konstanty Mazuruk at Marshall Space Flight Center and University of Alabama at Huntsville, Peter Goldreich at Caltech, Martha Pardavi-Horvath at John Washington University and the National Bureau of Standards in Washington DC, Yahya Rahmat-Samii at UCLA, Gary C. Gerlach at Orion Group, San Jose, CA, Guennadi A. Kouzaev at NTNU in Trondheim, Norway, Youri V. Shestopalov at Karlstad University, Sweden, Pablo M. Cincotta at IAFE, Buenos Aires, Argentina and to all participants of the seminars and conferences: the NASA Marshall Space Flight Center and the Huntsville Space Physics Colloquium; the Institute for Astronomy at the University of Hawaii; Astrophysics and the Space Research Center at the University of California in San Diego; University of California at Berkeley and Davis; the Institute of Astronomy and Physics, La Plata, Buenos Aires; Faculty of Engineering of Alexandria University, Egypt; the Progress In Electromagnetic Research Symposium (PIERS) organized by MIT; the 42nd - 50th SPIE Annual Meetings; the 30th Annual Meeting of the Division of Planetary Sciences of the American Astronomical Society and Pulkovo Astronomical Observatory of St. Petersburg, Russia.

\section{References}

[1] Maxwell J.C. 1859. On the Stability of the motion of Saturn's Rings. Monthly Notices of the Royal Astronomical Society. 19, 297-304; Maxwell J.C., Brush S.G., Everitt C.W.F., Garber E. (Eds.). 1983. Maxwell on Saturn's Rings. MIT Press, Cambridge, MA.

[2] Safronov V.S. 1969. Evolution of Protoplanetary Cloud and Formation of the Earth and Planets. Nauka, Moscow (Russian).

[3] Goldstein R.M., Morris G.A. 1973. Radar Observations of the Rings of Saturn. Icarus. 20, 249-283.

[4] Kaiser M.L., Desch V.D., Lecacheus A. 1981. Saturnian Kilometric Radiation: Statistical Properties and Beam
Geometry. Nature. 292, 731-733.

[5] Alfven H. 1981. Cosmic Plasma. Springer; 1983. Solar System History as Recorded in the Saturnian Ring Structure. Astrophysics and Space Science. 97, 79-94; 2011, Oct.-Dec. D. Talbott. The Plasma Universe of Hannes Alfven. Edgescience. 9, 5-10

[6] Brahic A. (Ed.). 1984. Planetary Rings. Toulouse, Copadeus; Greenberg R., Brahic A. (Eds.). 1984. Planetary Rings. University of Arizona Press, Tucson.

[7] Bliokh A.N., Yaroshenko V.V. 1991. "Spokes" in the Rings of Saturn. Nature. 4, 19-25 (Russian).

[8] Mendis D.A., Hill J.R., Ip W.H., Goertz C.K., and Grun E. 1984. Electrodynamics Processes in the Ring System of Saturn. Saturn. T. Gehrels, M. Mathews (Eds.). University of Arizona Press, Tucson. 546-589; 1994, Sept. Mendis D. A., Rosenberg M. Cosmic Dusty Plasma. Annual Review of Astronomy and Astrophysics. 32, 419-463

[9] Morrison D., Owen T.C. 2003. The Planetary System. Addison-Wesley Longman.

[10] N.N. Gor'kavyi, A.M. Fridman. 1994. Physics of the Planetary Rings: Celestial Mechanics of Continuous Medium. Nauka, Moscow (Russian).

[11] Rabinovich B.I. 1996. Magnetohydrodynamic of Rotating Vortex Rings with Magnetized Plasma. Doklady Physics. 351, 335-338; 1999. Rotating Plasma Ring in Gravitational and Magnetic Fields. Stability Problems. 367, 345-348 (Russian).

[12] Spilker L.J. (Ed.). 1997, Oct. The Cassini-Huygens Mission to Saturn and Titan. NASA SP-533. JPL, Caltech. Washington, D.C.

[13] Yokota T. 2001. Ring Simulation Experiment Using Fine Particle Plasmas. IEEE Trans. Plasma Science. 29, 279.

[14] P. K. Shukla, A. A. Mamun, and R. Bingham. 2003. Comment on Mach Cones and Magnetic Forces in Saturn's Rings. JETP Letters. 78, 2, 99-100.

[15] Rowan L., Sanchez-Lavega A., Gombosi T.I., Hansen K.S., Porco C.C. et al., Flasar F.M., Esposito L.W. et al., Gurnett D.A. et al., Waite J.H., Jr. et al., Young D.T. et al., Dougherty M.K. et al., Krimigis S.M. et al., Kempf S. et al. 2005, Febr. 25. Cassini at Saturn. Science. 307, 1222-1276;

[16] Burns J.A., Cuzzi J.N. 2006, June 23. Our Local Astrophysical Laboratory. Science. 312, 1753-1755;

[17] Dougherty M., Esposito L. and Krimigis T. (Eds.) 2009. Saturn from Cassini-Huygens. Springer, Dordrecht.

[18] Cuzzi J.N., J. A. Burns J.A., Charnoz S., Clark R.N., Colwell J.E., Dones L., Esposito L.W., Filacchione G., French R.G., Hedman M.M., Kempf S., Marouf E.A., Murray C.D., Nicholson P.D., Porco C.C., Schmidt J., Showalter M.R. , Spilker L.J., Spitale J.N., Srama R., Sremčević M., Tiscareno M.S., Weiss J. 2010, March 19. An Evolving View of Saturn's Dynamic Rings. Science. 327, 1470-1475.

[19] Salo H. 2011. Twisted Discs. Science. 332, 672; 2012. Simulating the Formation of Fine-Scale Structure in Saturn Rings. Progr. Theor. Phys. Supplement. 195, 48-67.

[20] Lyra W. 2013, July 11. Formation of Sharp Eccentric Rings 
in Debris Disks with Gas but without Planets. Nature. 184.

[21] Tiscareno M.S., Colin J. Mitchell C.S., Murray C.D., Di Nino D., Hedman M.M., Schmidt J., Burns J.A., Cuzzi J.N., Porco C.C., Beurle K., Evans M.W. 2013, April 26. Observations of Ejecta Clouds Produced by Impacts onto Saturn's Rings. Science. 340, 460-464

[22] Bednorz J.G., Müller K.A. 1986. Possible high Tc Superconductivity in the $\mathrm{Ba}-\mathrm{La}-\mathrm{Cu}-\mathrm{O}$ System. Z. Physik, B. 64, 189-193.

[23] Babushkina G.V., Kobelev L.Ya., Yakovlev E.N. Babushkin A.N. 1986. Superconductivity of Ice Under High Pressure. Physics of Solid State. 28, 3732-3734 (Russian).

[24] Côté M., Grossman J.C., Cohen M. L., Louie S.G. 1998. Electron-Phonon Interactions in Solid C36. Phys. Rev. Lett. $81,697$.

[25] Maeno N. 2004. Science of Ice. Moscow (Russian).

[26] Pospelov A.Yu., Tchernyi V.V. 1995. Electromagnetic Properties Material Forecast in the Planet Rings by the Methods of Functionally Physical Analysis. Proc. Intern. Scientific-Methodological Conf. on Innovative Design in Education, Techniques and Technologies. Volgograd State Technical University, Volgograd (Russian). 75-77.

[27] Pospelov A. Yu., Tchernyi V. V., Girich S.V. Planet's Rings: Super-diamagnetic Model and New Course of Investigations. 1997, July 27-Aug. 1. Proc. SPIE 42nd Annual Meet. San Diego, CA. Small spacecraft, Space Environments and Instrumentation Technologies. 3116. 117128; 1998, March 20-28. Planetary Rings: New Mission Concept. Paper № 132; Possible Explanation of the Planet's Rings Behavior in the Radio and mm-wave Range via Superdiamagnetic Model. Paper № 73. SPIE International Symposium on Astronomical Telescopes and Instrumentation. Kona, HW; 1998, July 20-23. Superdiamagnetic Model of Planetary Rings Behavior in the millimeter and submullimeter Range. Digest 3465 - 4th International Conference on MM and SMM Waves and Applications. San Diego, CA. Proc. SPIE 43rd Annual Intern. Sympos. San Diego, CA. 172-173.

[28] Girich S.V., Pospelov A.Yu., Tchernyi V.V. 1998, Oct. 11-16. Radar Data Explanation via Superdiamagnetic Model of the Saturn's Rings. Annual Report of AAS. 30th Meeting Division of Planetary Science. Madison, WI. Bulletin of the American Astronomical Society. 30. 1043.

[29] Tchernyi V.V., Pospelov A.Yu., Girich S.V. 1998. Studies on the Rings of Saturn. The Academy for Future Science. P.O. Box FE, Los Gatos, CA 95031;

[30] Pospelov A.Yu., Tchernyi V.V., Girich S.V. 1999, July 18-23. Anomalous Inversion of Polarization of Icy Satellites and Saturn's Rings: Superdiamagnetic Model. Proc. 44th SPIE Annual Meet. Denver, CO. Polarization: Measurements, Analysis and Remote Sensing II. 1999. 3754. 329-333; 2000, July 5-14. Are Saturn's Rings Superconducting? Progress in Electromagnetic Research Sympos. Cambridge, MA: MIT. 1158; 1999, Aug. 20. Are Saturn rings superconducting? University of Alabama, Huntsville. NASA Marshall Space Flight Center, Huntsville Space Physics Colloquium.
[31] Tchernyi V.V., Pospelov A.Yu. 2005. Possible Electromagnetic Nature of the Saturn's Rings: Superconductivity and Magnetic Levitation. Progress in Electromagnetic Research. PIER. 52, 277-299.

[32] Tchernyi V.V., Chensky E.V. 2005. Electromagnetic Background for Possible Magnetic Levitation of the Superconducting Rings of Saturn. Journal of Electromagnetic Waves and Applications. 19, 1997-2006.

[33] Tchernyi V.V., Chensky E.V. 2005. Movements of the Protoplanetary Superconducting Particles in the Magnetic Field of Saturn Lead to the Origin of Rings. Geoscience and Remote Sensing Letters-IEEE. 2, 4, 445-446; 2006. Corrections. IEEE GRSL. 3, 2.

[34] Tchernyi V.V. 2002, Aug. 7. Possible superconductivity of Saturn rings. University of Hawaii. Institute for Astronomy. Colloquia: Spring/Summer 2002; 2006. About Possible Role of Electromagnetism and Superconductivity for the Origin of Saturn Rings. Prikladnaya Fizika (Applied Physics). 5, 10-16 (Russian).

[35] Tchernyi V.V. (Cherny). 2006, Oct. 25-27. Possible Role of Superconductivity and Electromagnetism for the Origin of the Rings of Saturn. Proc. Intern. Conf. Fundamental Principles of Engineering Sciences. Devoted to 90-years Birthday of the Nobel Prize Winner A.M. Prokhorov. Moscow. 257-259. (Russian).

[36] Tchernyi V.V., Pospelov A.Yu. 2007. About Hypothesis of the Superconducting Origin of the Saturn's Rings. Astrophysics and Space Science. 307, 4, 347-356.

[37] Tchernyi (Cherny) V.V. 2009. Origin of the Saturn Rings: Electromagnetic Model of the Sombrero Rings Formation. Space Exploration Research. Denis J.H., Aldridge P.D. (Eds.). Nova Science Publishers, NY. Chapter 11, 261-275.

[38] Tchernyi V. V. 2009, Aug. 3-14. Discovery of Initial Formation (Origin) of the Sombrero Rings of Saturn: Role of Electromagnetism. International Astronomical Union. XXVII General Assembly. Rio de Janeiro, Brazil. IAU Sympos. No. 263. Icy Bodies in the Solar System. Aug. 6.

[39] Tchernyi (Cherny) V.V. 2013. Could Superconductivity Contribute to the Saturn Rings Origin? Journal of Modern Physics. 4, 6A, 17-23.

[40] Deutscher G., Azoulay M., Almog B., Deutscher B. 2011. ASTC Conf. Oct. 15-18. Quantum Levitation. http://www.youtube.com/watch?v=Ws6AAhTw7RA; http://www.ahigheru.org/25/post/2013/05/boaz-almog-usesquantum-physics-to-levitate-and-trap-objects-in-midair-callit-quantum-levitation.html\#.Uf1 Ii9IvmdI

[41] Tchernyi V.V., Kapranov S.V. 1999, July 31-Aug. 4. Contribution of superconductivity to possible interstellar propagation of organic molecules by electromagnetic way. Proc. 50th SPIE Annual Meeting. San Diego, CA.

[42] Tchernyi V.V., Kapranov S.V. 2005. Possible role of superconductivity for simplest life propagation within the interstellar space by electromagnetic force of magnetic levitation. Journal of Electromagnetic Waves and Applications. 19, 15, 1997-2006; Tchernyi V.V., Kapranov S.V. 2005. Astrobiology and Planetary Missions. Hoover R.B. et al. (Eds.). SPIE. 5906. 